\title{
Measurement of Regional Specific Lung Volume Change Using Respiratory-Gated PET of Inhaled ${ }^{13} \mathrm{~N}$-Nitrogen
}

Tyler J. Wellman ${ }^{1,2}$, Tilo Winkler ${ }^{2}$, Eduardo L.V. Costa ${ }^{2}$, Guido Musch², R. Scott Harris ${ }^{3}$, Jose G. Venegas ${ }^{2}$, and Marcos F. Vidal Melo ${ }^{2}$

${ }^{1}$ Department of Biomedical Engineering, Boston University, Boston, Massachusetts; ${ }^{2}$ Department of Anesthesia and Critical Care, Massachusetts General Hospital and Harvard Medical School, Boston, Massachusetts; and ${ }^{3}$ Pulmonary and Critical Care Unit, Massachusetts General Hospital and Harvard Medical School, Boston, Massachusetts

Regional specific lung volume change (sVol), defined as the regional tidal volume divided by the regional end-expiratory gas volume, is a key variable in lung mechanics and in the pathogenesis of ventilator-induced lung injury. Despite the usefulness of PET to study regional lung function, there is no established method to assess sVol with PET. We present a method to measure sVol from respiratory-gated PET images of inhaled ${ }^{13} \mathrm{~N}$-nitrogen $\left({ }^{13} \mathrm{NN}\right)$, validate the method against regional specific ventilation (sV), and study the effect of region-of-interest (ROI) volume and orientation on the sVol-sV relationship. Methods: Four supine sheep were mechanically ventilated (tidal volume $\mathrm{V}_{\mathrm{T}}=8 \mathrm{~mL} / \mathrm{kg}$, respiratory rate adjusted to normocapnia) at low $(n=2$, positive end-expiratory pressure $=0)$ and high $(n=2$, positive end-expiratory pressure adjusted to achieve a plateau pressure of $30 \mathrm{~cm} \mathrm{H}_{2} \mathrm{O}$ ) lung volumes. Respiratory-gated PET scans were obtained after inhaled ${ }^{13} \mathrm{NN}$ equilibration both at baseline and after a period of mechanical ventilation. We calculated sVol from ${ }^{13} \mathrm{NN}$-derived regional fractional gas content at end-inspiration $\left(F_{E I}\right)$ and end-expiration $\left(F_{E E}\right)$ using the formula $s \mathrm{VI}=\left(\mathrm{F}_{\mathrm{El}}-\mathrm{F}_{\mathrm{EE}}\right) /\left(\mathrm{F}_{\mathrm{EE}}\left[1-\mathrm{F}_{\mathrm{EI}}\right]\right) . \mathrm{s} \dot{\mathrm{V}}$ was computed as the inverse of the subsequent ${ }^{13} \mathrm{NN}$ washout curve time constant. ROls were defined by dividing the lung field with equally spaced coronal, sagittal, and transverse planes, perpendicular to the ventrodorsal, laterolateral, and cephalocaudal axes, respectively. Results: sVol-s $\dot{V}$ linear regressions for ROls based on the ventrodorsal axis yielded the highest $R^{2}$ (range, 0.71-0.92 for mean ROI volumes from 7 to $162 \mathrm{~mL}$ ), the cephalocaudal axis the next highest $\left(R^{2}=0.77-0.88\right.$ for mean ROI volumes from 38 to $\left.162 \mathrm{~mL}\right)$, and the laterolateral axis the lowest $\left(R^{2}=0.65-0.83\right.$ for mean ROI volumes from 8 to $162 \mathrm{~mL}$ ). ROls based on the ventrodorsal axis yielded lower standard errors of estimates of sVol from s $\dot{V}$ than those based on the laterolateral axis or the cephalocaudal axis. Conclusion: sVol can be computed with PET using the proposed method and is highly correlated with s.े. Errors in sVol are smaller for larger ROIs and for orientations based on the ventrodorsal axis.

Received Jul. 3, 2009; revision accepted Dec. 30, 2009.

For correspondence or reprints contact: Marcos F. Vidal Melo, Cardiac Anesthesia Group, Department of Anesthesia and Critical Care, Massachusetts General Hospital, Harvard Medical School, 55 Fruit St., Boston, MA 02114.

E-mail: mvidalmelo@partners.org

COPYRIGHT @ 2010 by the Society of Nuclear Medicine, Inc.
Key Words: positron emission tomography; respiratory-gated imaging techniques; ventilator-induced lung injury; lung volume measurements

J Nucl Med 2010; 51:646-653

DOI: 10.2967/jnumed.109.067926

$\mathbf{R}$ egional specific lung volume change ( $\mathrm{sVol}$ ), defined as regional tidal volume divided by regional end-expiratory (EE) gas volume, is a key variable in lung mechanics and in the pathogenesis of ventilator-induced lung injury. sVol provides information directly associated with tidal aeration and lung strain and allows for the estimation of regional elastance and ventilation $(1,2)$. Consequently, several methods have been developed to assess sVol or related quantities with CT (2-6) and MRI (7-10). PET allows for the assessment of regional lung shunt (11-13), ventilationto-perfusion ratios (14-19), and neutrophilic inflammation $(20,21)$. However, there is currently no method to quantify sVol with PET. Availability of such a technique would be valuable to investigate disease processes such as ventilatorinduced lung injury.

Recent CT studies have shown that sVol is linearly correlated with specific ventilation $(s \dot{V})$ in mechanically ventilated healthy supine sheep $(2,5)$. These studies indicate that measurements of sVol can be validated through their relationship with $s \dot{V}$. Indeed, regional $s \dot{V}$ depends directly on volume change ( $\mathrm{s} \dot{\mathrm{V}}=\Delta \mathrm{V} /[\mathrm{V} \cdot \Delta \mathrm{t}]$, where $\mathrm{V}$ is volume and $\mathrm{t}$ is time), and measurement of $s \dot{V}$ from ${ }^{13} \mathrm{~N}$-nitrogen $\left({ }^{13} \mathrm{NN}\right)$ washout with PET is well established $(14,16-18)$. Those CT studies also indicated that the accuracy of sVol depends on the volume of regions of interest (ROIs) due to registration effects. In addition, evidence of anisotropic lung expansion (22) and vertical gradients in lung volume and ventilation in supine humans and animals $(23,24)$ implies that sVol accuracy may also depend on ROI orientation, that is, if ROIs are delineated by dividing the field of view with equispaced coronal, sagittal, or transverse planes. 
Equilibration of inhaled ${ }^{13} \mathrm{NN}$ in the lungs leads to a distribution of ${ }^{13} \mathrm{NN}$ in proportion to regional gas volume. In this study, we present a method based on respiratorygated PET of equilibrated ${ }^{13} \mathrm{NN}$ to compute regional sVol from end-inspiratory (EI) and EE images of regional gas volume. The method is validated through comparisons against regional $s \dot{V}$ in mechanically ventilated sheep at different levels of lung inflation. We also study the influence of ROI size and orientation on the relationship between $s \dot{V}$ and $s \mathrm{Vol}$ and quantify the contributions of each Cartesian axis to total aeration and ventilation heterogeneity.

\section{MATERIALS AND METHODS}

\section{Animal Preparation}

The protocols of this study were approved by the Subcommittee on Research Animal Care of the Massachusetts General Hospital. Four sheep weighing $20.2 \pm 1.9 \mathrm{~kg}$ were premedicated with intramuscular ketamine $(5 \mathrm{mg} / \mathrm{kg})$ and midazolam $(1-2 \mathrm{mg} / \mathrm{kg})$. General anesthesia was induced with an intravenous bolus of propofol $(2-4 \mathrm{mg} / \mathrm{kg})$ and fentanyl $(5-10 \mu \mathrm{g} / \mathrm{kg})$ and maintained with a continuous infusion of propofol and fentanyl titrated to blood pressure and heart rate. Pancuronium $(0.1 \mathrm{mg} / \mathrm{kg})$ was used for muscle paralysis at induction and repeated every $90 \mathrm{~min}$. Femoral arterial and internal jugular venous lines were introduced for monitoring of blood pressures, blood sampling, and fluid infusion. Animals were anticoagulated with intravenous heparin (a 2,000-U bolus and 1,000 U hourly) to prevent blood clotting in the catheters and pulmonary embolism. A mainstream flow and pressure analyzer was connected to the endotracheal tube for measurement of tidal volume $\left(\mathrm{V}_{\mathrm{T}}\right)$ and airway pressure and computation of respiratory system compliance (Noninvasive Cardiopulmonary Management System, model 7300; Respironics). After preparation, sheep were transported to the PET suite.

\section{Experimental Protocols}

To study the relationship between $\mathrm{sVol}$ and $\mathrm{s} \dot{\mathrm{V}}$ under distinct physiologic conditions, we chose to analyze data on 4 sheep from 2 separate experimental protocols. For both protocols, $\mathrm{V}_{\mathrm{T}}$ was within recommended clinical values $\left(\mathrm{V}_{\mathrm{T}}=8 \mathrm{~mL} / \mathrm{kg}\right)$, with an inspired oxygen fraction of 0.3 , inspiratory-to-expiratory time ratio of $1: 2$, and respiratory rate between 20 and 28 breaths per minute to maintain normocapnia $\left(\mathrm{P}_{2} \mathrm{CO}_{2}=35-45 \mathrm{~mm} \mathrm{Hg}\right)$. The first protocol aimed to maximize regional aeration heterogeneity in healthy sheep by using zero end-expiratory pressure (ZEEP group, $n=2$ ) along a 16-h period. The second protocol aimed at simulating a clinical condition of mild lung injury with low-dose endotoxin $\left(10 \mathrm{ng} \cdot \mathrm{kg}^{-1} \cdot \mathrm{min}^{-1}\right)$ while maximizing homogeneity of lung aeration through the use of positive end-expiratory pressure (PEEP group, $n=2$ ). PEEP was adjusted according to a recently proposed strategy to maximize alveolar recruitment while limiting hyperinflation (25) by setting PEEP to the value at which a plateau pressure of $30-32 \mathrm{~cm} \mathrm{H}_{2} \mathrm{O}$ was achieved with constant $\mathrm{V}_{\mathrm{T}}$. These sheep were ventilated for $4 \mathrm{~h}$. For both protocols, PET images were obtained as described below at baseline and at the end of the mechanical ventilation period. Endotoxin infusion was initiated after acquisition of baseline images in the PEEP group.

\section{PET Protocol}

For all PET scans, sheep were positioned supine in the camera (Scanditronix PC4096; GE Healthcare), with the most caudal slice adjacent to the diaphragm dome. The camera collected 15 transverse slices of $6.5-\mathrm{mm}$ thickness over a $9.7-\mathrm{cm}$-long axial field, providing 3-dimensional data for an estimated $70 \%$ of the total lung volume (15). Transmission scans of $10 \mathrm{~min}$ were obtained using a uniform rotating pin source of ${ }^{68} \mathrm{Ge}$ to correct PET emission scans for tissue attenuation and to measure lung density (26). We acquired an initial emission scan of an intravenous injection of a ${ }^{13} \mathrm{NN}$-saline bolus $(\sim 1,000 \mathrm{MBq})$, consisting of 22 frames $(8 \times 2.5 \mathrm{~s}, 10 \times 10 \mathrm{~s}$, and $4 \times 30 \mathrm{~s})$. During the first $60 \mathrm{~s}$, the animal was kept in apnea to allow the tracer to diffuse from the blood and accumulate in alveolar airspaces. This scan provided images of pulmonary blood flow (15), which were used for the delineation of lung tissue in the field of view.

A previously described closed-circuit rebreathing system (18) was used to administer ${ }^{13} \mathrm{NN}$ gas. A period of $6-10$ min was allowed to ensure complete tracer equilibration in regions of low ventilation. Because of its low solubility in water and tissue (partition coefficient water/air $=0.018$ at $37^{\circ} \mathrm{C}$ ), inhaled ${ }^{13} \mathrm{NN}$ remains confined to alveolar airspaces and conducting airways, with the tracer concentration in a region after equilibration being proportional to the gas volume of that region.

Respiratory-gated emission scans of intrapulmonary ${ }^{13} \mathrm{NN}$ were obtained after tracer equilibration (mean activity, $303.4 \pm 92.5$ $\mathrm{kBq} / \mathrm{mL}$ ). Gating was based on thresholding of the inspiratory pressure signal using a custom LabView interface (version 8.2; National Instruments). Respiratory cycles were divided into 6 bins of equal duration, each bin containing counts from the same phase of the respiratory cycle. A scanning period of $5 \mathrm{~min}$ was used to acquire PET data over several breaths. EI and EE frames were selected from the resulting 6 gated image frames on the basis of maximum and minimum tracer activity in the field of view.

A washout scan of the inhaled ${ }^{13} \mathrm{NN}$ was acquired after the gated imaging to assess regional ventilation. Starting from tracer equilibrium in the lungs, an imaging sequence consisting of 16 frames $(12 \times 10 \mathrm{~s}$ and $4 \times 30 \mathrm{~s})$ was started. The washout was initiated at $60 \mathrm{~s}$ into the sequence by switching the breathing system to tracer-free gas.

PET images were reconstructed with a voxel size of $2 \times 2 \times 6.5$ $\mathrm{mm}$ using the standard convolution backprojection algorithm and were filtered with an in-plane, low-pass Hanning kernel, yielding an effective volumetric resolution of $1.1 \mathrm{~cm}^{3}$.

\section{Definition of Lung Fields for Analysis}

Volumetric masks of lung fields were delineated separately for the EI and EE frames by initially including all voxels whose ${ }^{13} \mathrm{NN}$ activity exceeded a threshold value of $40 \%-50 \%$ of the highest activity within the frame; including perfused but poorly aerated lung regions viewed in the ${ }^{13} \mathrm{NN}$ bolus infusion scan; and refining the masks manually to exclude the trachea, the 2 main bronchi, and partial-volume effects along the edges of the lung and near the heart. Caudal slices containing a significant volume of diaphragm were also excluded from the analysis.

\section{Computation of Regional Specific Volume Change}

Regional tidal sVol (defined as $\left[\mathrm{V}_{\mathrm{EI}}-\mathrm{V}_{\mathrm{EE}}\right] / \mathrm{V}_{\mathrm{EE}}$, where $\mathrm{V}_{\mathrm{EI}}=$ end-inspiratory gas volume and $\mathrm{V}_{\mathrm{EE}}=$ end-expiratory gas volume of a region) was computed as proposed by Fuld et al. (2): 


$$
s V_{o l}=\frac{F_{E I}-F_{E E}}{F_{E E}\left(1-F_{E I}\right)},
$$

where $\mathrm{F}_{\mathrm{EI}}$ and $\mathrm{F}_{\mathrm{EE}}$ are the mean fractional gas content $\left(\mathrm{F}_{\mathrm{gas}}\right)$ of a region at end-inspiration and end-expiration. $F_{\text {gas }}$ represents the fraction of the volume of a region occupied by gas. Equation 1 is based on $F_{\text {gas }}$ measurements rather than absolute volume measurements. This method of computing sVol is less sensitive to errors in precise delineation of ROIs than methods based on absolute volumes (2).

$\mathrm{F}_{\mathrm{EI}}$ and $\mathrm{F}_{\mathrm{EE}}$ were calculated from the gated ${ }^{13} \mathrm{NN}$ images. For this, the 6 gated image frames were averaged to produce a single image of mean regional tracer activity during the entire respiratory cycle. The corresponding transmission scan, which was obtained at mean lung volume, was processed as previously described (27) to construct an image of the average regional $\mathrm{F}_{\text {gas }}$. Finally, the scaling factor between ${ }^{13} \mathrm{NN}$ activity from gated emission scans and $\mathrm{F}_{\text {gas }}$ from the transmission scan was computed as the mean ratio of $F_{\text {gas }}$ to ${ }^{13} \mathrm{NN}$ activity in all voxels with $\mathrm{F}_{\text {gas }}$ greater than 0.5 . Such regions were chosen for their higher signal-to-noise ratio and to exclude partial-volume effects near lung edges.

ROIs for analysis were defined by dividing the EI and EE lung fields along 3 Cartesian axes (ventrodorsal, laterolateral, and cephalocaudal). ROIs were generated from 1-, 2-, or 3-dimensional grids based on equidistant divisions of those axes (Supplemental Fig. 1; supplemental materials are available online only at http://jnm.snmjournals.org). Thus, ROIs divided along the ventrodorsal axis (i.e., VD ROIs) were separated by equispaced coronal planes, along the laterolateral axis (i.e., laterolateral ROIs) were separated by equispaced sagittal planes, and along the cephalocaudal axis (i.e., cephalocaudal ROIs) were separated by equispaced transverse planes. For multidimensional division, equispaced planes along either 2 or 3 axes formed a grid of ROIs. The number of divisions along a given dimension ranged from 2 to the number required to produce ROIs of single-voxel thickness along that dimension. Larger numbers of divisions produced ROIs with smaller mean volumes. Corresponding ROIs in the EE and EI lungs fields were used to compute sVol.

\section{Calculation of Regional si}

Regional si was estimated by fitting the ${ }^{13} \mathrm{NN}$ kinetics during washout with a monoexponential curve:

$$
\mathrm{A}(\mathrm{t})=\mathrm{A}_{0} \cdot \mathrm{e}^{-\mathrm{s} \dot{\mathrm{V}} \cdot \mathrm{t}}
$$

where $\mathrm{A}(\mathrm{t})=$ tracer activity as a function of time, $\mathrm{A}_{0}=$ tracer activity at start of washout, $\mathrm{s} \dot{\mathrm{V}}=$ regional specific ventilation, and $\mathrm{t}=$ time.

Because the PET image data represent cumulative decay counts over specific time intervals, we used an integral-based approach and least-squares linear regression to estimate $\mathrm{A}_{0}$ and $\mathrm{s} \dot{\mathrm{V}}(28)$. Finally, to compare experiments with different respiratory rates (RR), perbreath specific ventilation $\left(\mathrm{s}_{\mathrm{B}}\right)$ was computed as:

$$
\mathrm{s} \dot{\mathrm{V}}_{\mathrm{B}}=\frac{\mathrm{s} \dot{\mathrm{V}}}{\mathrm{RR}}
$$

\section{Spatial Dependence of $F_{\text {gas }}$ and $s \dot{V}$}

We computed voxel-level $\mathrm{F}_{\text {gas }}$ from transmission scans and $\mathrm{s} \dot{\mathrm{V}}$ from washout scans (as described above) to assess their de- pendence on spatial position. A linear regression model (Eq. 4) was used to estimate the contributions of each Cartesian axis to the total variability in these voxel-level values.

$$
P_{i j k}=\mu+x_{i}+y_{j}+z_{k}+\varepsilon_{i j k} . \quad \text { Eq. } 4
$$

For each voxel value of $\mathrm{F}_{\text {gas }}$ or $\mathrm{s} \dot{\mathrm{V}}\left(\mathrm{P}_{\mathrm{ijk}}\right)$, the model accounts for contributions of the global mean $(\mu)$; specific position along the laterolateral $\left(\mathrm{x}_{\mathrm{i}}\right), \mathrm{VD}\left(\mathrm{y}_{\mathrm{j}}\right)$, and cephalocaudal $\left(\mathrm{z}_{\mathrm{k}}\right)$ axes; and a nonCartesian component $\left(\varepsilon_{\mathrm{ijk}}\right)$. The fractional contribution of each axis was computed as the variance related to position along that axis divided by the total variance. Finally, contributions from each axis were normalized such that their sum was equal to 1 .

\section{Sensitivity of Mean Regional $\mathrm{F}_{\text {gas }}$ to ROI Position}

Differences in tissue content of ROIs at end-inspiration and end-expiration could lead to errors in the assessment of sVol. To estimate the magnitude of this effect, we calculated mean $F_{\text {gas }}$ within ROIs as their boundaries were shifted similarly to displacements caused by lung motion or heterogeneous regional expansion. We divided the lung field of a ZEEP animal into 5 ROIs along each of the 3 Cartesian axes and examined mid lung ROIs. The mean $F_{\text {gas }}$ of each ROI was computed in its original position and after shifts of $6 \mathrm{~mm}$ along its axis of division. In addition, a cubic 18-mL ROI resulting from 3-dimensional division of the lung field was shifted along all 3 axes by $6 \mathrm{~mm}$.

\section{Statistical Methods}

Results are expressed as mean $\pm \mathrm{SD}$. The square of the Pearson product-moment correlation coefficient $\left(R^{2}\right)$ was used to quantify the correlations between $s$ Vol and $s \dot{V}_{B}$. ROIs with volume less than the mean $-1 \mathrm{SD}$ in each experiment, usually located at the edges of the lung, had an undue effect on $R^{2}$ calculations and were excluded from the analysis. Standard errors of estimates (SEEs) of $\mathrm{sVol}$ from $\mathrm{s} \dot{\mathrm{V}}_{\mathrm{B}}$ were computed to quantify and compare the predictive value of $\mathrm{s}$ ol for different ROI orientations and sizes. Distributions of the differences between predicted and measured sVol were compared using an ANOVA model including animal, time point, ROI volume, and orientation as independent variables. Values of $P$ less than 0.05 were considered significant.

\section{RESULTS}

Sheep mechanically ventilated with PEEP showed larger mean airway pressure and peak inspiratory pressure than those ventilated with ZEEP (Table 1). In all animals, respiratory system compliance decreased from baseline to the end of the experiment.

Respiratory gating yielded a sequence of frames along the breathing cycle consistent with changes in regional lung volume in all studied ROIs (Fig. 1). There were notable regional differences in gas content and tracer kinetics during ${ }^{13} \mathrm{NN}$ equilibrium and washout. The nondependent regions had the highest gas content throughout the respiratory cycle (Figs. 1A and 1C) and demonstrated the slowest washout rate (Figs. 1B and 1D). The dependent and middle regions had smaller gas contents, particularly at end-expiration (Figs. 1A and 1C) and exhibited faster washout rates than the nondependent regions (Figs. 1B and 1D). 
TABLE 1. Ventilator Settings and Respiratory Measurements

\begin{tabular}{|c|c|c|c|c|c|c|c|c|}
\hline \multirow[b]{2}{*}{$\begin{array}{l}\text { Ventilator } \\
\text { setting }\end{array}$} & \multicolumn{4}{|c|}{ ZEEP group } & \multicolumn{4}{|c|}{ PEEP group } \\
\hline & $\begin{array}{l}\text { Sheep } 1 \\
\text { baseline }\end{array}$ & $\begin{array}{c}\text { Sheep } 1 \\
\text { end }\end{array}$ & $\begin{array}{l}\text { Sheep } 2 \\
\text { baseline }\end{array}$ & $\begin{array}{c}\text { Sheep } 2 \\
\text { end }\end{array}$ & $\begin{array}{l}\text { Sheep } 3 \\
\text { baseline }\end{array}$ & $\begin{array}{c}\text { Sheep } 3 \\
\text { end }\end{array}$ & $\begin{array}{l}\text { Sheep } 4 \\
\text { baseline }\end{array}$ & $\begin{array}{c}\text { Sheep } 4 \\
\text { end }\end{array}$ \\
\hline $\mathrm{V}_{\mathrm{T}}(\mathrm{mL})$ & 193 & 176 & 157 & 165 & 186 & 185 & 168 & 159 \\
\hline PEEP $\left(\mathrm{cm} \mathrm{H}_{2} \mathrm{O}\right)$ & 0.0 & 0.0 & 0.0 & 0.0 & 13.3 & 14.5 & 18.9 & 18.3 \\
\hline $\mathrm{PIP}\left(\mathrm{cm} \mathrm{H} \mathrm{H}_{2} \mathrm{O}\right)$ & 13.4 & 22.0 & 15.9 & 19.4 & 29.0 & 35.0 & 32.2 & 37.0 \\
\hline $\mathrm{MAP}\left(\mathrm{cm} \mathrm{H} \mathrm{H}_{2} \mathrm{O}\right)$ & 3.0 & 4.5 & 3.2 & 3.7 & 16.6 & 19.2 & 22.1 & 22.2 \\
\hline $\mathrm{C}_{\mathrm{rs}}\left(\mathrm{mL} / \mathrm{cm} \mathrm{H} \mathrm{H}_{2} \mathrm{O}\right)$ & 17.1 & 9.1 & 12.9 & 10.4 & 17.9 & 14.6 & 16.5 & 14.8 \\
\hline
\end{tabular}

The relationship between $s \dot{V}_{B}$ and $\mathrm{sVol}$ in 10 VD ROIs showed a strong linear correlation for all time points in both groups (Fig. 2). $R^{2}$ for all experiments ranged from 0.65 to 0.93 , with an average of $0.79 \pm 0.11$. All linear correlations were significant at $P$ less than 0.01 . The mean slope of the regression lines was $2.63 \pm 0.45$, and the $y$-intercept was $0.08 \pm 0.07$. The average ROI volume among both ZEEP and PEEP experiments for 10 VD ROIs was $49 \mathrm{~mL}$. Experiments with ZEEP (Fig. 2A) showed a larger range of $s \dot{V}_{B}$ and sVol than those with PEEP (Fig. 2B) and also tended to have higher $y$-intercepts $(P<0.06)$. This presence of positive $y$-intercepts dependent on PEEP level is consistent with a theoretical prediction of the relationship between $\mathrm{S}_{\mathrm{B}}$ and sVol (Supplemental Appendix).

In ROIs divided along only 1 axis, the $R^{2}$ values between $s \dot{V}_{B}$ and sVol were dependent on both the ROI volume and the orientation (Fig. 3A). For all orientations, $R^{2}$ increased with ROI size. Removal of volumetric outliers was particularly important to improve $R^{2}$ in VD and laterolateral ROIs for volumes less than $50 \mathrm{~mL}$. VD ROIs yielded the highest
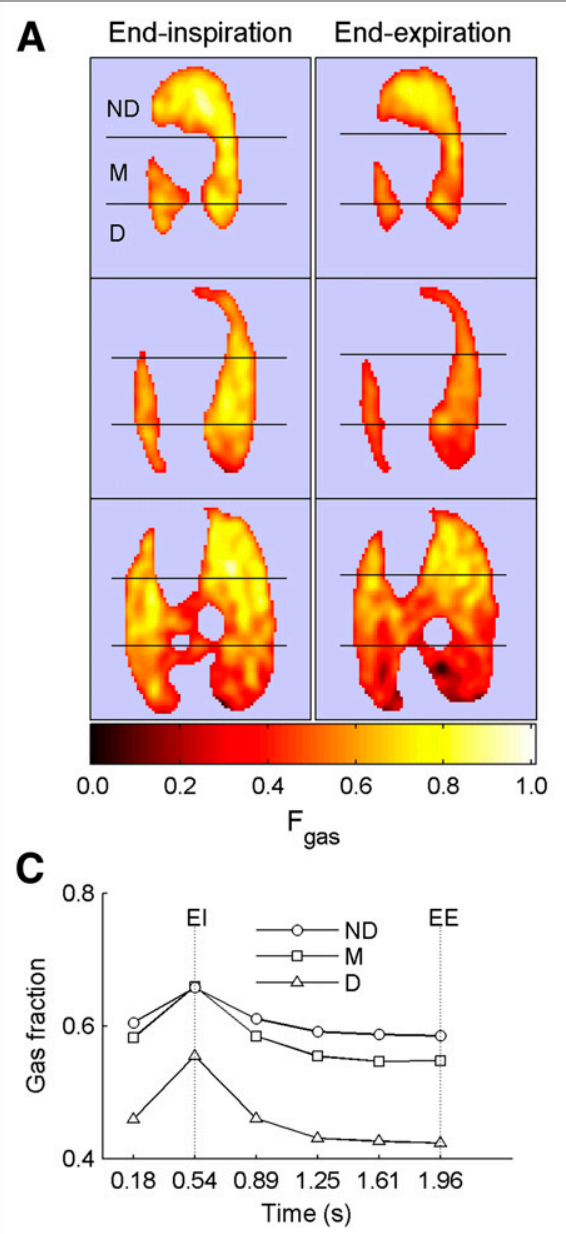
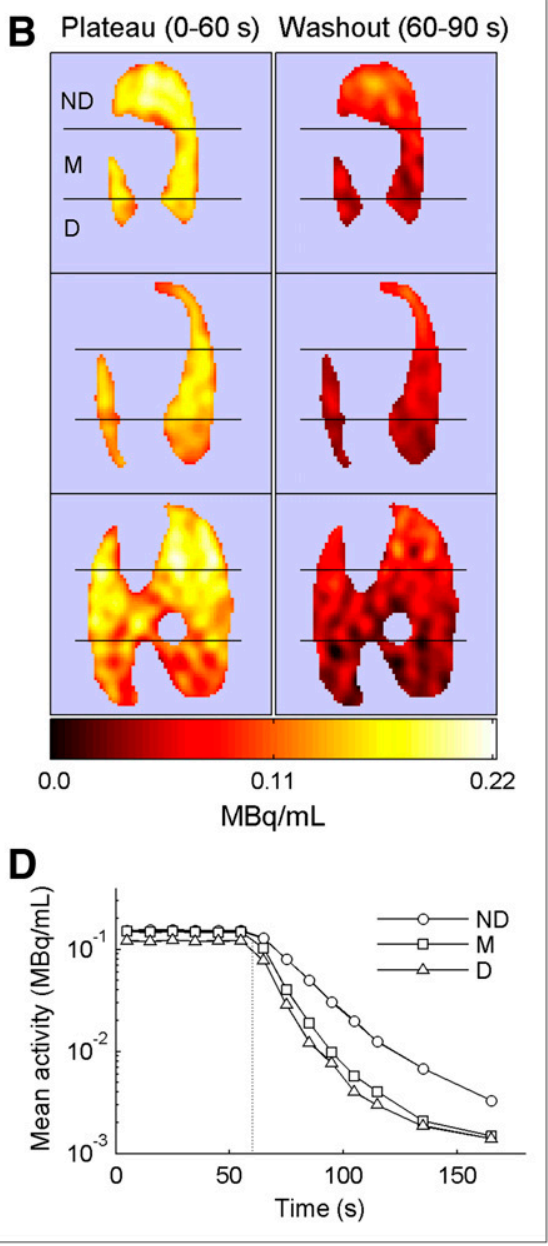

FIGURE 1. PET scans and tracer kinetics in animal ventilated with zero EE pressure and tidal volume of $8 \mathrm{~mL} /$ $\mathrm{kg}$. Hot color scale is used in all images, with black equaling zero regional activity and white equaling maximal regional activity. Left side in image corresponds to left side in animal. (A) El and EE frames of regional gas volume from respiratory-gated ${ }^{13} \mathrm{NN}$ PET scans, shown in 3 transverse slices of lung from apex (top) to base (bottom). Lungs are divided into 3 isogravitational ROls: nondependent (ND), middle (M), and dependent (D). (B) Images of ${ }^{13} \mathrm{NN}$ content at mean lung volume during ${ }^{13} \mathrm{NN}$ equilibrium (plateau) and during first $30 \mathrm{~s}$ of washout. Rate of decrease in tracer activity in dependent and middle regions is higher than that in nondependent regions. (C) Respiratorygated gas fractions in nondependent, middle, and dependent regions. Maximum gas fraction occurs at El, whereas minimum occurs at EE. (D) ${ }^{13} \mathrm{NN}$ washout kinetics for same lung regions. Vertical dotted line indicates washout start. 
FIGURE 2. Relationship between $s \dot{\mathrm{V}}_{\mathrm{B}}$ and $\mathrm{sVol}$ in 10 ventrodorsal ROls for each sheep at baseline (empty symbols) and after ventilation (filled symbols). Note different axis scales for experiments with ZEEP (A) and experiments with PEEP (B), with each sheep in these groups represented by different symbol. $R^{2}$ ranged from 0.65 to 0.93 , with average of $0.79 \pm 0.11$. For ZEEP experiments, $s \dot{\mathrm{V}}_{\mathrm{B}}$ and $\mathrm{sVol}$ values, ranges, and $y$-intercepts tended to be higher than for PEEP experiments.
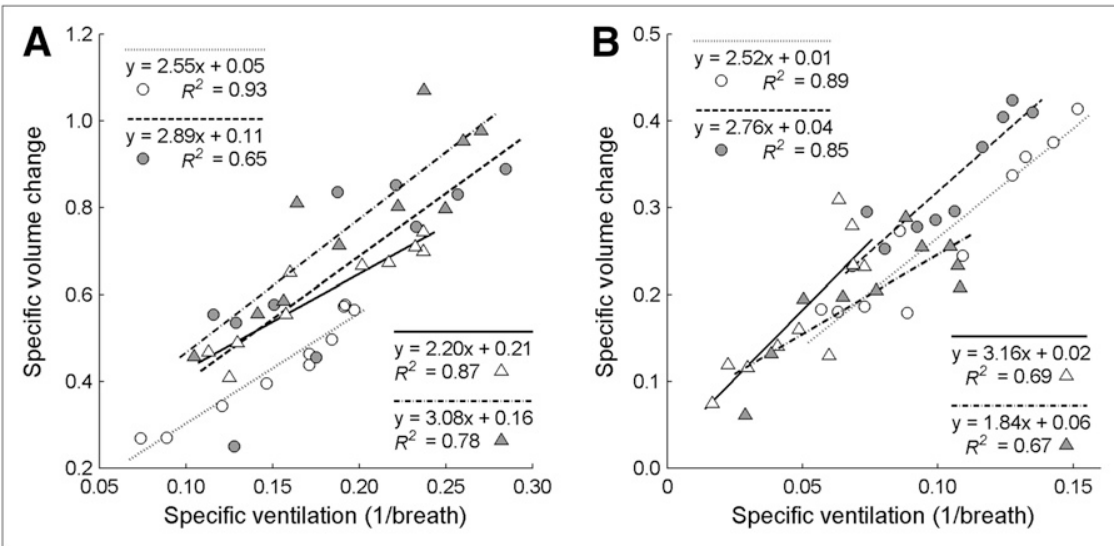

$R^{2}$ ( $>0.85$ for all volumes), cephalocaudal ROIs yielded the next highest $R^{2}$ ( $>0.76$ for all volumes), and laterolateral ROIs produced the lowest $R^{2}$, which were still greater than 0.73 even for the smallest mean volumes.

The dependence of $R^{2}$ on ROI size and orientation was also present when ROIs were generated by multidimensional division of the field of view along either 2 or 3 axes (Fig. 3B). $R^{2} \mathrm{~s}$ were highest when VD divisions were combined with divisions along the other axes, with $R^{2}$ greater than 0.64 for all such combinations at mean ROI volumes greater than or equal to $15 \mathrm{~mL}$. Combining divisions along the laterolateral and cephalocaudal axes yielded $R^{2}$ greater than 0.50 for mean ROI volumes greater than $17 \mathrm{~mL}$. Below $25 \mathrm{~mL}, R^{2}$ seemed to decrease more rapidly with further reduction in ROI volume.

VD ROIs yielded significantly lower differences between measured $\mathrm{sVol}$ and $\mathrm{sVol}$ predicted from $\mathrm{sVol}-\mathrm{sV}_{\mathrm{B}}$ regressions than laterolateral $(P<0.001)$ and cephalocaudal $(P<0.001)$ ROIs. This result is consistent with the SEEs of $\mathrm{sVol}$ from $s \dot{\mathrm{V}}_{\mathrm{B}}$ for different ROI orientations, which are lowest for VD ROIs at all ROI volumes (Fig. 4). For all orientations, SEEs generally decreased with increase in mean ROI volume. Overall, SEEs at all volumes were below 0.10 for $\mathrm{VD}, 0.13$ for laterolateral, and 0.11 for cephalocaudal ROIs.
The contributions of the different axes to total variance in voxel-level $F_{\text {gas }}$ and $s \dot{V}$ were studied separately for experiments with ZEEP and PEEP (Supplemental Fig. 2). For both conditions, position along the VD axis exhibited the most influence on both variables, with the laterolateral axis having the next largest influence. For $\mathrm{F}_{\mathrm{gas}}$, PEEP acted to homogenize the influences of the different axes. In contrast, the contribution of the VD axis to total $\mathrm{S} \dot{\mathrm{V}}$ variance appeared to be even larger for PEEP than for ZEEP experiments.

Shifting ROI boundaries along their axes of division resulted in small changes in mean regional $\mathrm{F}_{\text {gas }}$ (Fig. 5). Relative changes in $\mathrm{F}_{\text {gas }}$ when shifting boundaries along only 1 axis were under $2.7 \%$ for all cases. In addition, shifting the cubic ROI resulted in a maximum relative change in $\mathrm{F}_{\text {gas }}$ of only $2.8 \%$.

\section{DISCUSSION}

The following are the 2 main results of this study in supine mechanically ventilated sheep. First, PET-based regional measurements of lung specific volume change derived from respiratory-gated images of equilibrated inhaled ${ }^{13} \mathrm{NN}$ are strongly correlated with specific ventilation computed from the subsequent ${ }^{13} \mathrm{NN}$-washout. Second, correlation between regional specific lung volume change and specific ventilation
FIGURE 3. Coefficients of determination $\left(R^{2}\right)$ between $\mathrm{sV}_{\mathrm{B}}$ and $\mathrm{sVol}$ in ROls generated by either unidimensional $(A)$ or multidimensional (B) division of lung field along ventrodorsal (VD), laterolateral (LL), or cephalocaudal (CC) axes. Gray symbols in A for VD and LL axes represent values of $R^{2}$ before removal of volumetric outliers. Effect of removing outliers is larger for smaller ROls. $R^{2}$ generally decreased with reduction in mean $\mathrm{ROI}$ volume. In B, all combinations including divisions along VD axis have similarly high $R^{2}(>0.64)$ at $15 \mathrm{~mL}$ and above.
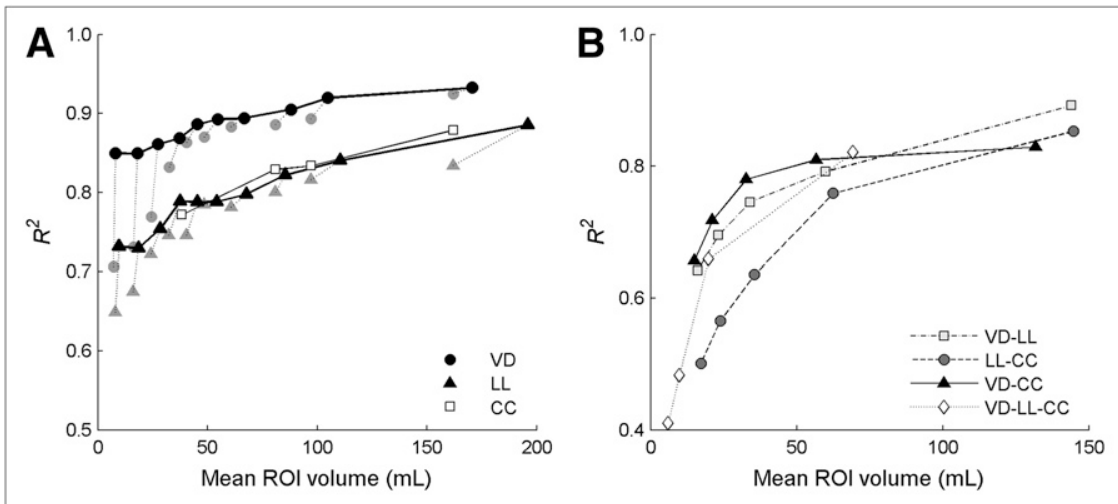


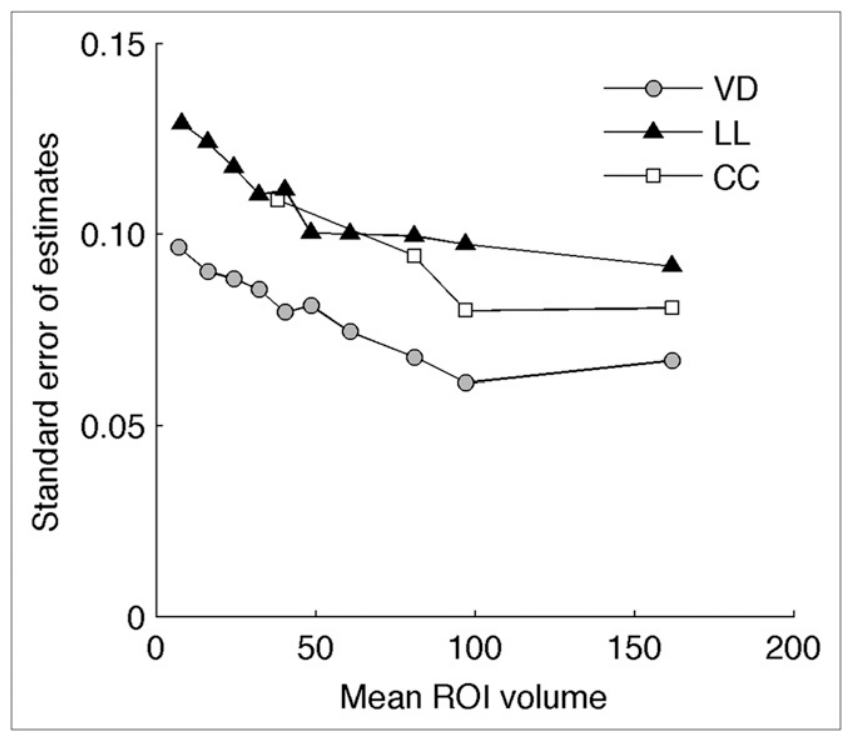

FIGURE 4. SEEs of $s$ ol from $s$ ol-s $\dot{V}_{B}$ regression lines. SEEs were lower for ventrodorsal (VD) than for laterolateral (LL) and cephalocaudal (CC) orientations. For all orientations, SEEs increased for smaller ROI volumes.

is highest, and SEEs of $s$ Vol from $s \dot{V}_{B}$ are lowest, for larger ROIs and for ROIs divided along the VD axis.

Lung tissue strain is a fundamental variable in pulmonary mechanics and an important promoter of lung injury and dysfunction $(20,21,29,30)$. Consequently, CT and MRI techniques were developed to assess regional volume changes (2-10). Respiratory-gated PET has been applied to image thoracic tumors (31-33). However, there has been limited use in the assessment of lung function (16), limiting, among others, accurate estimates of lung ventilation (14). Our results support the use of respiratory-gated PET to assess sVol. PET methods to quantify regional function $(11-19,23,26,34)$ and inflammation $(20,21)$ can be combined with the proposed method, providing inherent coregistration and a valuable tool to study lung dysfunction and injury during mechanical ventilation.
$\mathrm{sVol}$ and $\mathrm{s} \dot{\mathrm{V}}_{\mathrm{B}}$ assess different quantities. sVol measures tidal changes in mean regional gas fraction over multiple breaths and primarily depends on true changes in regional gas volume. It is also affected by regional blood volume, lung motion, partial-volume effects, and the quality of coregistration between EI and EE images. $s \dot{\mathrm{V}}_{\mathrm{B}}$ is also mainly influenced by regional volume changes. Additionally, it is affected by partial-volume effects and by factors unique to $s \dot{V}_{B}$ including serial and parallel dead space, intraregional gas mixing, and pendelluft. A lower sVol$s \dot{V}_{\mathrm{B}}$ correlation could result from any of the listed factors influencing each measurement independently. Because the major factor contributing to both $s$ Vol and $s \dot{V}_{B}$ is the regional tidal volume, high $s$ Vol-s $\dot{\mathrm{V}}_{\mathrm{B}}$ correlations reflect a systematic assessment by both variables of regional tidal volume. Because correlation coefficients are affected by range, we also computed SEEs to quantify the dispersion of the predictions of $\mathrm{sVol}$ with $\mathrm{s}_{\mathrm{B}}$. Those results confirmed the trends found with correlation coefficients in the comparisons among ROI orientations and sizes.

Importantly, CT measurements of sVol based on the same principles used in this study were correlated with direct assessments of $\mathrm{sVol}$ obtained with parenchymal markers (2). These direct validation data, combined with our results, provide a strong indication that $\mathrm{sVol}$ and $\mathrm{s}_{\mathrm{B}}$ measured with PET are both useful to assess regional lung volume changes during mechanical ventilation. Because $\mathrm{sVol}$ is the variable most directly associated with regional volume changes, it is the preferred method. When respiratory gating is unavailable, $s \dot{V}_{B}$ provides information about regional volume changes, if factors unique to $s \dot{V}_{B}$ can be assumed to be unimportant.

Our measurements of $R^{2}$ were computed from data derived from all animals and time points, providing a measure of the global correlation between $s \dot{\mathrm{V}}_{\mathrm{B}}$ and $\mathrm{sVol}$. The use of data from multiple animals provides a large range of values in both $s \dot{V}_{B}$ and $s \mathrm{Vol}$ for each ROI configuration, such that the values of $R^{2}$ are indicative of the predictive power of one variable from the other. This is supported by the fact that the SEEs of the $s$ Vol-s $\dot{V}_{B}$

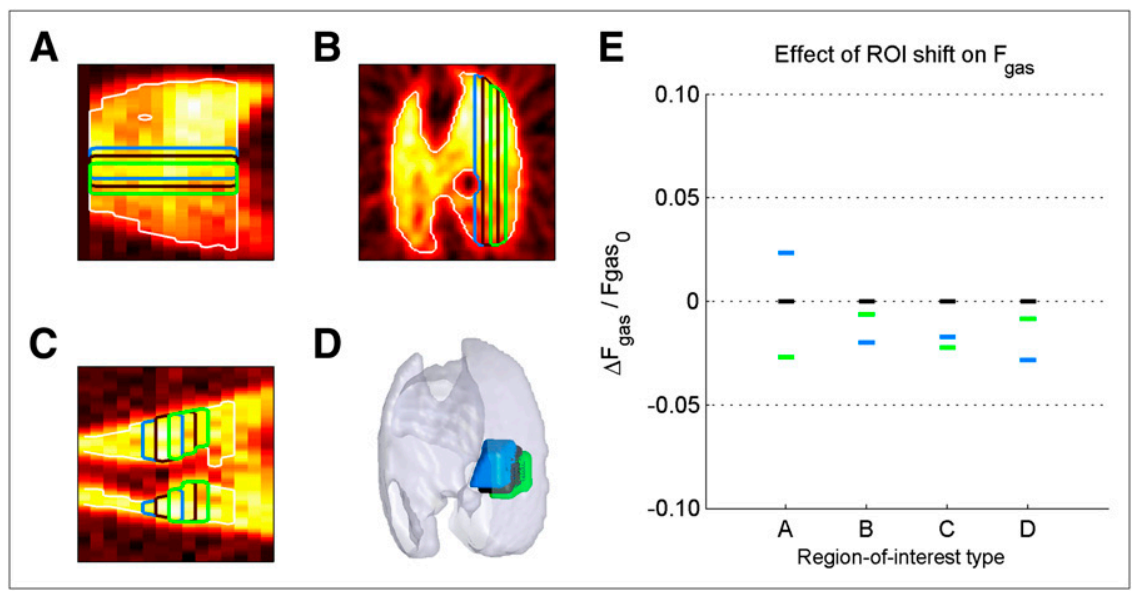

FIGURE 5. Effect of ROI boundary shifts on mean regional $F_{\text {gas. }}$ ( $\left.A-D\right)$ ROls used to compute mean $F_{\text {gas }}$, divided along the ventrodorsal axis ( $A$, sagittal plane), laterolateral axis $(B$, transverse plane), cephalocaudal axis (C, coronal plane), and combining all directions ( $D$, 3-dimensional view). (E) Mean regional gas fractions for each ROI type in A-D (represented by respective colors) demonstrated small changes in $F_{\text {gas }}$ as result of ROI boundary shifts. 
regressions, which are not affected by the range of the variables, are consistent with the data on $R^{2}$, showing smaller errors for VD and larger ROIs.

sVol-s $\dot{\mathrm{V}}_{\mathrm{B}}$ correlations were higher for larger ROI volumes. Such results are compatible with the reduction of imaging noise on estimates of $\mathrm{F}_{\text {gas }}$ and $\mathrm{s}_{\mathrm{B}}$ with larger regions. In addition, larger ROIs tend to reduce the effect of coregistration error between gated EI and EE images and between the gated images and the $\mathrm{s}_{\mathrm{B}}$ measurements at mean lung volume.

The orientation of ROIs was also an important determinant of the sVol-s $\dot{\mathrm{V}}_{\mathrm{B}}$ correlation. VD ROIs lead to larger $R^{2}$ and smaller SEEs than laterolateral or cephalocaudal ROIs of the same size. In fact, $R^{2}$ values greater than 0.85 were found for VD ROIs less than $10 \mathrm{~mL}$. This result is consistent with our finding of a high contribution of the $\mathrm{VD}$ axis to total heterogeneity in regional $\mathrm{F}_{\text {gas }}$ and $\mathrm{s} \dot{\mathrm{V}}$. Our results expand previous findings $(2,23,24)$ by suggesting that VD variability in $F_{\text {gas }}$ and $s \dot{V}$ predominates in supine sheep even when submitted to high levels of PEEP and that a substantial fraction of total sVol heterogeneity is also associated with the VD axis.

The low sensitivity of sVol measurements to lung motion is an important characteristic of the presented method. Because current PET cameras do not allow for highresolution tracking of anatomic tissue references as done with CT (2-5), registration errors between images at different lung volumes due to lung motion could be problematic. However, the method to compute sVol used in our study relies on the assessment of regional $\mathrm{F}_{\mathrm{gas}}$, which varies smoothly with lung position. We showed that registration errors do not significantly compromise the measurements of $F_{\text {gas }}$ and sVol. The same advantage has been demonstrated for CT assessment of sVol (2). Errors in mean $F_{\text {gas }}$ could become important, with significant density heterogeneity at length scales similar to the ROI size.

Another important feature of the method is that $\mathrm{sVol}$ results from dynamic gated scans acquired over several minutes of steady-state breathing. These scans represent the time-averaged regional gas content better than a single-breath measurement, relevant in the presence of significant interbreath variability. Multibreath acquisition of gated images may also improve the $s \mathrm{Vol}-\mathrm{s} \dot{\mathrm{V}}_{\mathrm{B}}$ correlation, because $\mathrm{s} \dot{\mathrm{V}}_{\mathrm{B}}$ is measured over several breathing cycles. Another factor improving $\mathrm{sVol}-\mathrm{s} \dot{\mathrm{V}}_{\mathrm{B}}$ correlations is that both measurements derive from ${ }^{13} \mathrm{NN}$ imaging. In contrast, CT measurements use lung density to compute sVol and xenon as a radiodense contrast agent to assess ventilation $(2,4,5)$. In this case, density changes may be due to either changes in xenon concentration or movement of tissue components into and out of the field of view, resulting in a potential increase in measurement error (35).

Removal of outlier ROIs (volume $<$ mean -1 SD) had a notable effect on improving $R^{2}$ at low mean ROI volumes. On average, these ROIs corresponded to a negligible fraction of the total lung field $(\sim 0.5 \%)$. Such ROIs were usually at the edge of the lung field and were significantly influenced by partial-volume effects because of their small size $(<2 \mathrm{~mL})$.

We fit a nonzero intercept line to the experimental sVol$s \dot{V}_{B}$ data, in contrast to a previous study (2), because the presence of a dead space leads to a positive intercept in the $s$ Vol-s $\dot{\mathrm{V}}_{\mathrm{B}}$ regression (Supplemental Appendix). Intercepts were larger in the sVol-s $\dot{\mathrm{V}}_{\mathrm{B}}$ lines for the ZEEP than for the PEEP group. PEEP leads to a larger increase in regional lung volume than in dead space (36), decreasing the dead space-to-lung volume ratio. Simulations describing the effect of this decrease on the $s$ Vol-s $\dot{V}_{\mathrm{B}}$ relationship substantiated this finding (Supplemental Appendix), indicating that our PET measurements of $s \mathrm{Vol}$ and $s \dot{V}_{B}$ are accurate enough to quantify details in the $s$ Vol-s $\dot{V}_{B}$ relationship.

\section{CONCLUSION}

We presented a technique for the assessment of regional lung expansion using respiratory-gated PET of inhaled ${ }^{13} \mathrm{NN}$ and compared its measurements with those derived from the subsequent ${ }^{13} \mathrm{NN}$ washout. Measurements of regional volume changes using gated imaging were highly correlated with per-breath specific ventilation computed from inhaled ${ }^{13} \mathrm{NN}$ washout, suggesting that both of those variables provide reliable assessments of regional tidal lung volume change in the studied conditions. Errors in the estimates of regional lung volume change depend on ROI size and orientation and decrease with increasing volume and with the orientation of the ROIs used for analysis, being smallest for VD ROIs.

\section{ACKNOWLEDGMENTS}

We thank the staff of the Division of Nuclear Medicine at Massachusetts General Hospital and, in particular, Steven B. Weise for expert technical assistance in image acquisition and reconstruction. We also thank Hui Zheng for assistance with statistical analysis. This work was supported by the National Heart, Lung, and Blood Institute grant 5R01-HL086827.

\section{REFERENCES}

1. Bellardine Black CL, Hoffman AM, Tsai LW, et al. Impact of positive end-expiratory pressure during heterogeneous lung injury: insights from computed tomographic image functional modeling. Ann Biomed Eng. 2008;36:980991.

2. Fuld MK, Easley RB, Saba OI, et al. CT-measured regional specific volume change reflects regional ventilation in supine sheep. J Appl Physiol. 2008;104: 1177-1184.

3. Pan Y, Kumar D, Hoffman EA, et al. Estimation of regional lung expansion via 3D image registration. Proc SPIE. 2005;57466:453-464.

4. Reinhardt JM, Christensen GE, Hoffman EA, Ding K, Cao K. Registrationderived estimates of local lung expansion as surrogates for regional ventilation. Inf Process Med Imaging. 2007;20:763-774.

5. Reinhardt JM, Ding K, Cao K, Christensen GE, Hoffman EA, Bodas SV. Registration-based estimates of local lung tissue expansion compared to xenon CT measures of specific ventilation. Med Image Anal. 2008;12:752-763.

6. Simon BA. Regional ventilation and lung mechanics using X-ray CT. Acad Radiol. 2005;12:1414-1422. 
7. Chen Q, Mai VM, Bankier AA, Napadow VJ, Gilbert RJ, Edelman RR. Ultrafast MR grid-tagging sequence for assessment of local mechanical properties of the lungs. Magn Reson Med. 2001;45:24-28.

8. Gee J, Sundaram T, Hasegawa I, Uematsu H, Hatabu H. Characterization of regional pulmonary mechanics from serial magnetic resonance imaging data. Acad Radiol. 2003;10:1147-1152.

9. Napadow VJ, Mai V, Bankier A, Gilbert RJ, Edelman R, Chen Q. Determination of regional pulmonary parenchymal strain during normal respiration using spin inversion tagged magnetization MRI. J Magn Reson Imaging. 2001;13:467-474.

10. Voorhees A, An J, Berger KI, Goldring RM, Chen Q. Magnetic resonance imaging-based spirometry for regional assessment of pulmonary function. Magn Reson Med. 2005;54:1146-1154.

11. O'Neill K, Venegas JG, Richter T, et al. Modeling kinetics of infused 13NNsaline in acute lung injury. J Appl Physiol. 2003;95:2471-2484.

12. Musch G, Bellani G, Vidal Melo MF, et al. Relation between shunt, aeration, and perfusion in experimental acute lung injury. Am J Respir Crit Care Med. 2008;177:292-300.

13. Willey-Courand DB, Harris RS, Galletti GG, Hales CA, Fischman A, Venegas JG. Alterations in regional ventilation, perfusion, and shunt after smoke inhalation measured by PET. J Appl Physiol. 2002;93:1115-1122.

14. Richard JC, Janier M, Lavenne F, et al. Quantitative assessment of regional alveolar ventilation and gas volume using ${ }^{13} \mathrm{~N}-\mathrm{N} 2$ washout and PET. J Nucl Med. 2005;46:1375-1383.

15. Vidal Melo MF, Layfield D, Harris RS, et al. Quantification of regional ventilation-perfusion ratios with PET. J Nucl Med. 2003;44:1982-1991.

16. Treppo S, Mijailovich SM, Venegas JG. Contributions of pulmonary perfusion and ventilation to heterogeneity in $\dot{\mathrm{V}}_{\mathrm{A}} / \dot{\mathrm{Q}}$ measured by PET. J Appl Physiol. 1997;82:1163-1176.

17. Venegas JG, Schroeder T, Harris S, Winkler RT, Melo MF. The distribution of ventilation during bronchoconstriction is patchy and bimodal: a PET imaging study. Respir Physiol Neurobiol. 2005;148:57-64.

18. Vidal Melo MF, Harris RS, Layfield D, Musch G, Venegas JG. Changes in regional ventilation after autologous blood clot pulmonary embolism. Anesthesiology. 2002;97:671-681.

19. Vidal Melo MF, Harris RS, Layfield JD, Venegas JG. Topographic basis of bimodal ventilation-perfusion distributions during bronchoconstriction in sheep. Am J Respir Crit Care Med. 2005;171:714-721.

20. Schroeder T, Vidal Melo MF, Musch G, Harris RS, Venegas JG, Winkler T. Modeling pulmonary kinetics of 2-deoxy-2-[ ${ }^{18}$ F $]$ fluoro-D-glucose during acute lung injury. Acad Radiol. 2008;15:763-775.

21. Musch G, Venegas JG, Bellani G, et al. Regional gas exchange and cellular metabolic activity in ventilator-induced lung injury. Anesthesiology. 2007; 106:723-735.
22. Mitzner W, Fallica J, Bishai J. Anisotropic nature of mouse lung parenchyma. Ann Biomed Eng. 2008;36:2111-2120.

23. Musch G, Layfield JD, Harris RS, et al. Topographical distribution of pulmonary perfusion and ventilation, assessed by PET in supine and prone humans. J Appl Physiol. 2002;93:1841-1851.

24. Pelosi P, D'Andrea L, Vitale G, Pesenti A, Gattinoni L. Vertical gradient of regional lung inflation in adult respiratory distress syndrome. Am J Respir Crit Care Med. 1994;149:8-13.

25. Mercat A, Richard JC, Vielle B, et al. Positive end-expiratory pressure setting in adults with acute lung injury and acute respiratory distress syndrome: a randomized controlled trial. JAMA. 2008;299:646-655.

26. Rhodes CG, Wollmer P, Fazio F, Jones T. Quantitative measurement of regional extravascular lung density using positron emission and transmission tomography. J Comput Assist Tomogr. 1981;5:783-791.

27. Harris RS, Willey-Courand DB, Head CA, et al. Regional VA, Q, and VA/Q during PLV: effects of nitroprusside and inhaled nitric oxide. J Appl Physiol. 2002;92:297-312.

28. Foss SD. A method of exponential curve fitting by numerical integration. Biometrics. 1970;26:815-821.

29. Chiumello D, Carlesso E, Cadringher P, et al. Lung stress and strain during mechanical ventilation for acute respiratory distress syndrome. Am J Respir Crit Care Med. 2008;178:346-355.

30. Gattinoni L, Carlesso E, Cadringher P, Valenza F, Vagginelli F, Chiumello D. Physical and biological triggers of ventilator-induced lung injury and its prevention. Eur Respir J Suppl. 2003;47:15s-25s.

31. Wang Y, Baghaei $\mathrm{H}, \mathrm{Li} \mathrm{H}$, et al. A simple respiration gating technique and its application in high-resolution PET camera. IEEE Trans Nucl Sci. 2005;52:125129.

32. Nehmeh SA, Erdi YE, Ling CC, et al. Effect of respiratory gating on quantifying PET images of lung cancer. $J$ Nucl Med. 2002;43:876-881.

33. Nehmeh SA, Erdi YE, Rosenzweig KE, et al. Reduction of respiratory motion artifacts in PET imaging of lung cancer by respiratory correlated dynamic PET: methodology and comparison with respiratory gated PET. J Nucl Med. 2003; 44:1644-1648.

34. Richard JC, Le Bars D, Costes N, et al. Alveolar recruitment assessed by positron emission tomography during experimental acute lung injury. Intensive Care Med. 2006;32:1889-1894.

35. Simon BA, Marcucci C, Fung M, Lele SR. Parameter estimation and confidence intervals for Xe-CT ventilation studies: a Monte Carlo approach. J Appl Physiol. 1998;84:709-716.

36. Maisch S, Reissmann H, Fuellekrug B, et al. Compliance and dead space fraction indicate an optimal level of positive end-expiratory pressure after recruitment in anesthetized patients. Anesth Analg. 2008;106:175-181. 